\section{IL-23 inhibitor guselkumab shows promise for PsA}

The results of the DISCOVER-1 and DISCOVER-2 trials - two phase III trials assessing the IL-23 inhibitor guselkumab as a treatment for psoriatic arthritis (PsA) - suggest that guselkumab is efficacious in the treatment of PsA and could provide an additional treatment option for patients with this disease.

"Evidence from preclinical models suggested that the IL-23T helper $17\left(\mathrm{~T}_{\mathrm{H}} 17\right)$ pathway has a predominant role in the pathogenesis of inflammatory disease including psoriasis and PsA," explains Atul Deodhar, corresponding author on the DISCOVER-1 trial. "These latest results confirm the potential benefit of targeting the IL-23 cytokine in the treatment of PsA," continues corresponding author on the DISCOVER-2 trial, Philip Mease.

Guselkumab selectively targets IL-23 by binding to its p19 subunit. This drug is already approved for the treatment of patients with moderate-to-severe psoriasis and, in a previous phase II proof-ofconcept study, improved signs and symptoms of PsA, leading to these latest two trials.

The DISCOVER-1 trial aimed to assess guselkumab in patients with a broad range of baseline levels of disease activity. The enrolled patients had active PsA (at least three swollen and three tender joints and CRP $\geq 0.3 \mathrm{mg} / \mathrm{dL}$ ) and included patients who had an inadequate response or intolerance of standard treatment (including apremilast, DMARDs or NSAIDs) or TNF inhibition.

"Allocating approximately $30 \%$ of the study participants to individuals previously treated with one or two TNF inhibitors enabled the evaluation of guselkumab in patients who require a different mechanism of action to safely and effectively treat all aspects of their disease," reports Deodhar.

By contrast, the larger DISCOVER-2 trial aimed to assess guselkumab in biologic-naive patients with active PsA (at least five swollen joints, at least five tender joints and CRP $\geq 0.6 \mathrm{mg} / \mathrm{dL}$ ), despite standard therapies.

"In both trials, two guselkumab dosing regimens were studied: the regimen that is approved to treat psoriasis and that showed efficacy in the phase II proof-of-concept study (guselkumab $100 \mathrm{mg}$ given every 8 weeks), as well as a more frequent dosing interval (guselkumab $100 \mathrm{mg}$ given every 4 weeks) to evaluate whether higher serum concentrations would afford greater efficacy in PsA," explains Deodhar.

The primary end point (the proportion of patients who achieved an ACR20 response ( $\geq 20 \%$ improvement in ACR criteria) at week 24) was met for both guselkumab dosing regimens in both trials. In the DISCOVER- 1 trial, $59 \%$ of patients in the every 4 weeks group and 52\% of patients in the every 8 weeks group had an ACR20 response at week 24 compared with only $22 \%$ of patients in the placebo group. Similarly, $64 \%$ of patients in both the every 4 weeks group and the every 8 weeks group had an ACR20 response in the DISCOVER-2 trial, compared with $33 \%$ of patients in the placebo group. The percentage differences versus placebo were statistically significant for all the treatment groups across both studies $(P<0.0001)$.
"The most notable findings were that guselkumab was shown to benefit multiple clinical domains of PsA in both of these study populations," says Mease. Treatment with guselkumab (using either regimen) improved joint and skin symptoms as well as physical function and health-related quality of life in both trials. Furthermore, in the DISCOVER-2 trial, the 4-week regimen could inhibit progression of structural damage versus placebo, as assessed by changes in the PsA-modified van der Heijde-Sharp (vdHS) score at week 24.

Analysis of pooled data from DISCOVER-1 and DISCOVER-2 also revealed that a higher proportion of patients in either treatment group had clinically resolved dactylitis and enthesitis at week 24 than in the placebo group.

Both guselkumab regimens had a favourable safety profile that was consistent with the safety profile observed in the treatment of patients with psoriasis. These trials are being extended (DISCOVER-1 for 1 year and DISCOVER-2 for 2 years) to provide additional data on the efficacy and safety of guselkumab. "Further assessments of combined data from both trials are also planned, including integrating guselkumab safety data across trials and assessing the effects of guselkumab on PsA axial disease," says Deodhar.

"An important question to be addressed is whether IL-23 inhibition can benefit axial inflammation in patients with PsA, a clinical domain not typically addressed in PsA trials," states Mease. "Data on patients with PsA spondylitis were collected in the DISCOVER programme and will be presented at upcoming meetings."

Jessica McHugh

ORIGINAL ARTICLES Deodhar, A. et al. Guselkumab in patients with active psoriatic arthritis who were biologic-naive or had previously received TNFa inhibitor treatment (DISCOVER-1): a double-blind, randomised, placebo-controlled phase 3 trial. Lancet 395 , 1115-1125 (2020) | Mease, P. J. et al. Guselkumab in biologic-naive patients with active psoriatic arthritis (DISCOVER-2): a double-blind, randomised, placebo-controlled phase 3 trial. Lancet 395, 1126-1136 (2020) 